\title{
Mind maps
}

Arindam Basu

1 University of Canterbury

Mind maps refer to the process of drawing visualisation of concepts using a diagram where a central concept branches out with subtopics from centre outwards. Mind maps were first proposed by T ony Buzan and subsequently have been widely used in a number of different contexts. 